\title{
Transformação Integral e Problemas Inversos aplicados à Estimativa de Parâmetros em um Modelo 2D de Transporte de Sedimentos Não-Coesivos
}

\author{
Ademilton Luiz Rodrigues de Souza ${ }^{1}$ \\ Centro Federal de Educação Tecnológica Celso Suckow da Fonseca, CEFET/RJ \\ Diego Campos Knupp ${ }^{2}$ \\ Pedro Paulo Gomes Watts Rodrigues ${ }^{3}$ \\ Antônio José da Silva Neto ${ }^{4}$ \\ Universidade do Estado do Rio de Janeiro, Instituto Politécnico - Nova Friburgo, RJ, Brasil
}

Resumo. Neste trabalho apresenta-se uma abordagem de solução híbrida analítico-numérica com a Técnica da Transformada Integral Generalizada (GITT) para solução de um problema bidimensional transiente do transporte de sedimentos não-coesivos. Foram aplicados filtros analíticos para homogeneizar os contornos e melhorar a convergência. A equação foi transformada em um sistema acoplado de equações diferenciais parciais unidimensional que foi resolvido numericamente empregando recursos do Software Wolfram Mathematica ${ }^{\circledR} 10$. Os resultados obtidos pela GITT se mostraram coerentes e apresentaram ganhos computacionais, o que é desejável para a solução do problema inverso de identificação de parâmetros hidrodinâmicos e de transporte, utilizando o método de Monte Carlo via Cadeias de Markov.

Palavras-chave. Transporte de Sedimentos, GITT, Problemas Inversos, Inferência Bayesiana, Cadeias de Markov.

\section{Introdução}

No decorrer dos anos houve uma crescente preocupação com o aumento da concentração de poluentes em corpos d' água, que se originou principalmente pelo crescimento industrial e populacional desordenado. O movimento de sedimentos e de poluentes representa um dos problemas mais comuns enfrentados pela população mundial, e por isso o conhecimento e a caracterização do comportamento dos sedimentos em meio hídrico se tornam essenciais para a proteção do meio ambiente, manutenção dos canais de navegação, construção de obras hidráulicas, conservação do solo e dos recursos hídricos disponíveis [11].

Encontram-se na literatura vários trabalhos com propostas de solução para versões simplificadas da equação de advecção-dispersão que simula o transporte de sedimentos não-coesivos [10,12]. Por outro lado, alguns autores [7] obtiveram uma solução numérica

\footnotetext{
${ }^{1}$ alrsouza@iprj.uerj.br

${ }^{2}$ diegoknupp@iprj.uerj.br

${ }^{3}$ pwatts@iprj.uerj.br

4 ajsneto@iprj.uerj.br
} 
da versão 3D desse problema utilizando volumes finitos, mas com um custo computacional elevado, devido principalmente à complexidade das condições de contorno e do meio.

Dentre as diferentes metodologias que surgiram para combinar a flexibilidade dos métodos numéricos com a robustez dos métodos analíticos destaca-se a técnica da Transformada Integral Generalizada - GITT (Generalized Integral Transform Technique) que é um método híbrido, capaz de gerar soluções computacionais eficientes para diversos problemas a priori não transformáveis ou não solucionáveis numericamente [3,12].

Neste trabalho é realizada a estimação de parâmetros hidrodinâmicos e de transporte utilizando inferência Bayesiana e uma solução híbrida do modelo 2D-vertical de transporte de sedimentos não-coesivos empregando a GITT e a plataforma Wolfram Mathematica ${ }^{\circledR}$.

\section{Problema Direto 2D de Transporte de Sedimentos Não- Coesivos}

A concentração de sedimentos não-coesivos para cada classe granulométrica pode ser representada pela seguinte equação de advecção-dispersão [7], e respectivas condições de contorno e inicial [8]:

$$
\begin{gathered}
\frac{\partial C(x, z, t)}{\partial t}+U(z) \frac{\partial C(x, z, t)}{\partial x}+\frac{\partial\left(W-W_{s}\right) C(x, z, t)}{\partial z}=D_{x} \frac{\partial^{2} C(x, z, t)}{\partial x^{2}}+D_{z} \frac{\partial^{2} C(x, z, t)}{\partial z^{2}} \\
C(0, z, t)=C_{0}, \quad 0 \leq z \leq L_{z}, \quad t>0 \\
\frac{\partial C\left(L_{x}, z, t\right)}{\partial x}=0, \quad 0 \leq z \leq L_{z}, \quad t>0 \\
W_{s} C\left(x, L_{z}, t\right)+D_{z} \frac{\partial C\left(x, L_{z}, t\right)}{\partial z}=0, \quad 0 \leq x \leq L_{x}, \quad t>0 \\
W_{s} C_{a}+D_{z} \frac{\partial C(x, 0, t)}{\partial z}=D-E, \quad 0 \leq x \leq L_{x}, \quad t>0 \\
C(x, z, 0)=C_{1}, \quad 0 \leq x \leq L_{x}, \quad 0 \leq z \leq L_{z}
\end{gathered}
$$

onde $U(z)$ e $W$ representam, respectivamente, a velocidade do escoamento nas direções $x$ e $z, W_{s}$ é a velocidade de queda da partícula de sedimento, $D_{x}$ e $D_{z}$ são os coeficientes de dispersão da concentração longitudinal e vertical, respectivamente, $L_{x}$ e $L_{z}$ são os comprimentos longitudinal e vertical do domínio de interesse, $C_{0}$ representa a emissão contínua de um poluente na fronteira, na posição $x=0, C_{1}$ é a concentração inicial de sedimentos ao longo de todo o domínio, $C_{a}$ é uma concentração de referência no leito do escoamento em condições de equilíbrio e $D$ e $E$ são os fluxos de deposição e erosão de sedimentos, respectivamente.

Para acelerar e otimizar a convergência da técnica GITT, o problema pode ser simplificado com a aplicação da função filtro $C_{f}(z)=\operatorname{Exp}\left[-\frac{W_{s}}{D_{z}} z\right] / W_{s}$ (considerando $W=0$ ), obtida analiticamente e utilizada para homogeneizar as condições de contorno em $z$ pela expressão $C(x, z, t)=C_{f}(z)+C^{*}(x, z, t)$, onde $C^{*}(x, z, t)$ é a solução do problema filtrado obtida através da técnica GITT, como uma expansão em autofunções. 


\subsection{Problema de Autovalor e Problema Transformado}

A solução do problema filtrado é obtida por um esquema de transformação parcial, apenas na direção transversal, resultando em um problema transformado formado por um sistema de equações diferenciais parciais unidimensional. Em consequência, o seguinte problema de autovalor do tipo Sturm-Liouville associado é proposto:

$$
\begin{gathered}
D_{z} \frac{d^{2} \psi_{i}(z)}{d z^{2}}+\beta_{i}^{2} \psi_{i}(z)=0 \\
W_{s} C_{a}+D_{z} \frac{d \psi_{i}(0)}{d z}=0=W_{s} \psi_{i}\left(L_{z}\right)+D_{z} \frac{d \psi_{i}\left(L_{z}\right)}{d z}
\end{gathered}
$$

O problema apresentado permite definir o seguinte par de transformação integral:

$$
\begin{array}{cr}
\bar{C}_{i}(x, t)=\int_{0}^{L_{z}} \widetilde{\psi}_{i}(z) C^{*}(x, z, t) d z & \text { (transformada) } \\
C^{*}(x, z, t)=\sum_{i=1}^{\infty} \widetilde{\psi}_{i}(z) \bar{C}_{i}(x, t) & \text { (inversa) }
\end{array}
$$

onde $\widetilde{\psi}_{i}(z)=\frac{\psi_{i}(z)}{\sqrt{N_{i}}}$ são as autofunções normalizadas com $N_{i}=\int_{0}^{L_{z}} \widetilde{\psi}(z)^{2} d z$.

O problema filtrado pode então ser transformado através do operador $\int_{0}^{L_{z}} \widetilde{\psi}_{i}(z)() d$.$z para$ obter o seguinte problema transformado com respectivas condições inicial e de contorno:

$$
\begin{gathered}
\frac{\partial \overline{C_{i}}(x, t)}{\partial t}+\sum_{j=1}^{\infty} \frac{d \bar{C}_{j}(x, t)}{d x} \int_{0}^{L_{z}} \widetilde{\psi_{i}}(z) \widetilde{\psi_{j}}(z) U(z) d z+\left(W-W_{s}\right) \sum_{j=1}^{\infty} \bar{C}_{j}(x, t) \int_{0}^{L_{z}} \widetilde{\psi_{i}}(z) \frac{d \widetilde{\psi_{j}}(z)}{d z} d z= \\
=D_{x} \frac{\partial^{2} \overline{C_{i}}(x, t)}{\partial x^{2}}-\beta_{i}^{2} \overline{C_{i}}(x, t) \\
\overline{C_{i}}(x, 0)=\int_{0}^{L_{z}} \widetilde{\psi_{i}}(z)\left(C_{1}-C_{f}(z)\right) d z \\
\overline{C_{i}}(0, t)=\int_{0}^{L_{z}} \widetilde{\psi_{i}}(z)\left(C_{0}-C_{f}(z)\right) d z
\end{gathered}
$$

O sistema formado pelas Equações (4a-d) é resolvido numericamente para os potenciais transformados $\overline{C_{i}}(x, t)$, de modo que a fórmula de inversão, Equação (3b), pode ser empregada para obtenção da solução para o potencial filtrado, $C^{*}(x, z, t)$. 


\section{Problema Inverso}

Neste trabalho tem-se por objetivo obter estimativas para os parâmetros $D_{x}, D_{z}, \bar{U}$ e $W_{s}$ presentes nas Equações (1a-e) com uma abordagem de problemas inversos.

No tratamento de problemas inversos surgem diferentes fatores que contribuem para as incertezas na identificação de parâmetros que podem ser consideradas e contornadas ao se empregar a abordagem Bayesiana, onde os parâmetros são analisados como variáveis aleatórias. As vantagens desta abordagem são a possibilidade de incluir informações $a$ priori e incorporá-las em um contexto formal de decisão, bem como o tratamento explícito das incertezas e a habilidade de assimilar novas informações em contextos adaptativos [2].

A solução do problema inverso pela abordagem Bayesiana consiste em se obter a probabilidade a posteriori $\left(P_{\text {post }}\left(\boldsymbol{\beta} \mid \boldsymbol{Z}_{e}\right)\right)$, com o emprego do teorema de Bayes, tal que:

$$
P_{\text {post }}\left(\boldsymbol{\beta} \mid \boldsymbol{Z}_{e}\right)=P\left(\boldsymbol{Z}_{e} \mid \boldsymbol{\beta}\right) P_{p r}(\boldsymbol{\beta}) / P\left(\boldsymbol{Z}_{e}\right)
$$

onde $P_{p r}(\boldsymbol{\beta})$ é a probabilidade a priori, $P\left(\boldsymbol{Z}_{e}\right)$ é a função de densidade de probabilidade marginal e $P\left(\boldsymbol{Z}_{e} \mid \boldsymbol{\beta}\right)$ é a verossimilhança obtida pela Equação (6) que descreve a probabilidade de se encontrar os dados experimentais $\boldsymbol{Z}_{e}$, sabendo a resposta real e desconhecida do problema físico $(\boldsymbol{Z}(\boldsymbol{\beta}))$ e a variância $(\boldsymbol{V})$ dos dados experimentais [9]:

$$
P\left(\boldsymbol{Z}_{e} \mid \boldsymbol{\beta}\right)=\frac{1}{\sqrt{\operatorname{det} \boldsymbol{V}(2 \pi)^{n_{e}}}} \exp \left[-\frac{1}{2}\left(\boldsymbol{Z}_{e}-\boldsymbol{Z}^{m}\right)^{T} \boldsymbol{V}^{-1}\left(\boldsymbol{Z}_{e}-\boldsymbol{Z}^{m}\right)\right]
$$

onde $n_{e}$ representa o número de dados experimentais considerados no problema.

Em problemas mais complexos a informação a priori nem sempre pode ser representada por uma distribuição normal ou mesmo uniforme, o que torna muito complicado a obtenção de uma distribuição de probabilidade a posteriori. Nesses casos é necessário o emprego de técnicas de amostragem para simular as amostras de distribuição a posteriori e inferir medidas de tendência central e de dispersão desta distribuição [6]. Dentre essas técnicas de amostragem destaca-se o método de Monte Carlo via Cadeias de Markov (MCMC). A ideia essencial do MCMC é simular uma sequência aleatória de distribuições a posteriori convergente para uma distribuição estacionária e de interesse do problema inverso.

\section{Resultados}

Para a análise e comprovação da efetividade dos métodos de solução do problema direto e inverso foram considerados os seguintes valores $C_{0}=50 \mathrm{mg} / \mathrm{l}, C_{1}=50 \mathrm{mg} / \mathrm{l}$, $C_{a}=100 \mathrm{mg} / \mathrm{l}, D-E=0,1 \mathrm{~kg} / \mathrm{m}^{2} \mathrm{~s}$ e $W=0$. Para a velocidade longitudinal $(U(z))$ foi traçado um perfil logarítmico em função de $z$ e da velocidade média do escoamento $(\bar{U})$.

Para a solução do problema direto foram considerados os valores $W_{s}=0,013 \mathrm{~m} / \mathrm{s}$, $\bar{U}=1 \mathrm{~m} / \mathrm{s}$, e coeficientes de dispersão $D_{x}=0,25 \mathrm{~m}^{2} / \mathrm{s}$ e $D_{z}=0,006 \mathrm{~m}^{2} / \mathrm{s}$ obtidos pelas fórmulas empíricas estabelecidas, respectivamente, por [5] e [4].

Na Tabela 1 é apresentado o comportamento da convergência da solução à medida que se aumenta a ordem de truncamento $(N)$ da série na Equação (3b), de modo a atender o nível de precisão da solução e manter o mínimo esforço computacional. Observa-se que 
os resultados convergiram para pelo menos dois dígitos significativos, demonstrando que apenas 5 termos da série já é suficiente para obter uma boa convergência do modelo.

Tabela 1: Análise de convergência da solução em relação à ordem de truncamento $(z=0,5 \mathrm{~m})$.

\begin{tabular}{rrrrrrr}
\hline \hline $\mathrm{X}$ & $\mathrm{N}=2$ & $\mathrm{~N}=5$ & $\mathrm{~N}=10$ & $\mathrm{~N}=20$ & $\mathrm{~N}=50$ & $\mathrm{~N}=100$ \\
\hline \hline & & & $t=100 \mathrm{~s}$ & & & \\
0 & 47.93984 & 49.55828 & 49.90111 & 50.02409 & 49.99622 & 50.00093 \\
40 & 42.85569 & 42.81924 & 42.84078 & 42.84237 & 42.85687 & 42.86311 \\
80 & 37.08368 & 36.99077 & 37.00014 & 37.00086 & 37.00966 & 37.01231 \\
120 & 34.82856 & 34.71409 & 34.72044 & 34.72027 & 34.71984 & 34.71854 \\
160 & 34.83832 & 34.72336 & 34.73027 & 34.73046 & 34.73033 & 34.73026 \\
200 & 34.83837 & 34.72358 & 34.73027 & 34.73045 & 34.73047 & 34.73048 \\
\hline \hline
\end{tabular}

Visando a solução do problema inverso de identificação dos parâmetros $D_{x}, D_{z}, W_{s}$ e $\bar{U}$, a primeira etapa consistiu em realizar uma análise de sensibilidade para estudar o efeito que a variação destes parâmetros pode ocasionar nos resultados. Esta análise contribui significativamente na formulação e solução de problemas inversos pois permite, entre outras coisas, a escolha de dados experimentais que resultem em melhores estimativas para os parâmetros de interesse [1].

Na Figura 1 são apresentadas as curvas para cada coeficiente de sensibilidade obtidas numericamente através de uma aproximação por diferenças finitas centradas, considerando a expressão $X_{j} \cong\left(C\left(\beta_{j}+\epsilon_{j}\right)-C\left(\beta_{j}-\epsilon_{j}\right)\right) / 2 \epsilon_{j}$ com uma pequena variação $\epsilon_{j}$ para cada parâmetro desconhecido $j$. Observou-se que as maiores variações para cada coeficiente de sensibilidade foram encontrados nos primeiros 150 segundos. Diante disso, para a análise do problema inverso, foram gerados dados experimentais sintéticos até 150 segundos com intervalo de 2 segundos, considerando-se erros aditivos de uma distribuição normal com média zero e desvio padrão de $5 \%$ da concentração inicial. Observa-se também que os valores de sensibilidade para $D_{x}$ e $\bar{U}$ se mostraram relativamente pequenos em comparação com os demais valores, o que pode causar uma impossibilidade de estimação desses parâmetros. Para contornar esse problema, foi considerada uma priori informativa para os parâmetros $D_{x}$ e $\bar{U}$ segundo uma distribuição normal com médias $0,25 \mathrm{~m}^{2} / \mathrm{s}$ e $1,0 \mathrm{~m} / \mathrm{s}$, respectivamente, e desvio padrão de $10 \%$ de cada média.

Os resultados do MCMC estão apresentados na Figura 2, onde observa-se os histogramas e a convergência das cadeias de Markov para os valores médios $D_{x}=0,2509 \mathrm{~m}^{2} / \mathrm{s}$, $D_{z}=0,00622 \mathrm{~m}^{2} / \mathrm{s}, \bar{U}=0,9661 \mathrm{~m} / \mathrm{s}$ e $W_{s}=0,0134 \mathrm{~m} / \mathrm{s}$. Para alcançar a convergência foram considerados uma cadeia de 100000 estados e aquecimento de 50000 estados.

\section{Conclusões}

A metodologia utilizada permitiu, através do problema inverso, estimar parâmetros hidrodinâmicos e de transporte, em geral, de difícil medição, consequentemente possibilitando a observação de comportamentos esperados para a concentração de sedimentos não-coesivos em corpos d'água naturais. Os resultados obtidos mostraram a evolução do 


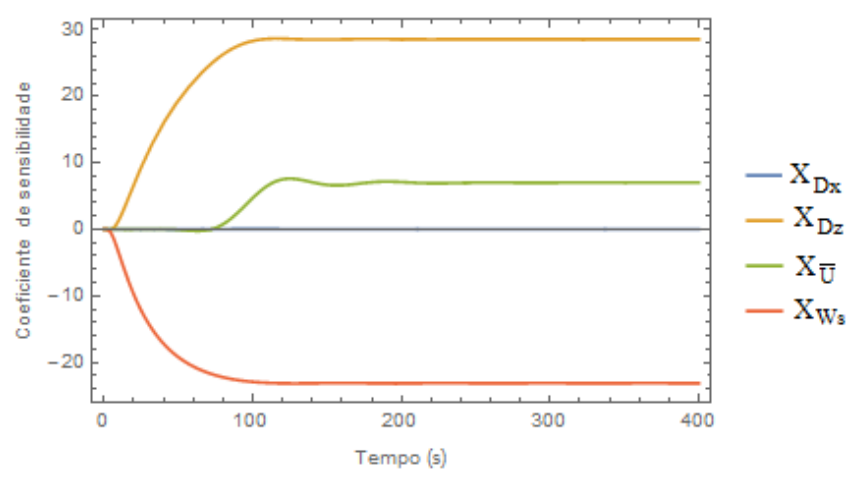

Figura 1: Coeficientes de sensibilidade para os parâmetros $D_{x}, D_{z}, \bar{U}$ e $W_{s}$.

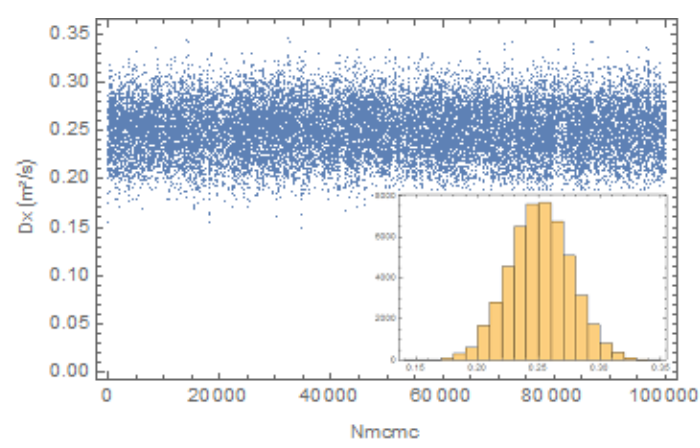

(a) $D_{x}$

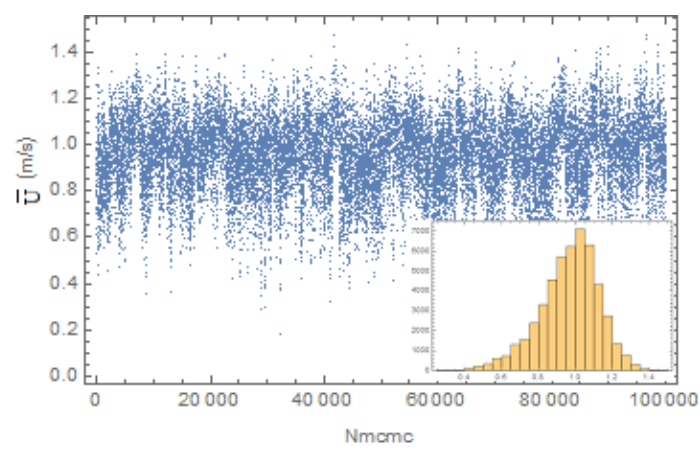

(c) $\bar{U}$

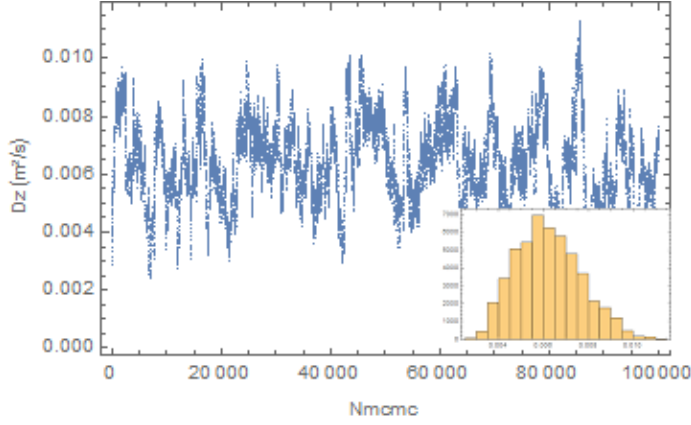

(b) $D_{z}$

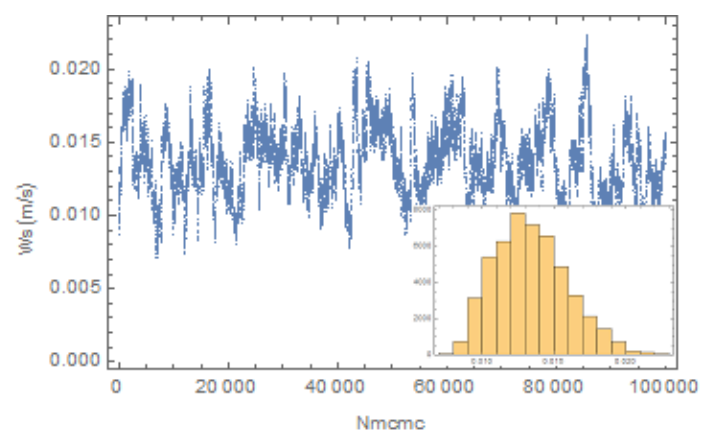

(d) $W_{s}$

Figura 2: Evolução das Cadeias de Markov.

perfil de concentração de sedimentos em suspensão, e o decaimento do campo de concentração nos casos de baixa disponibilidade de sedimentos. A utilização da técnica GITT na solução do problema proposto se mostrou plausível e rápida, como era desejável para a viabilidade da solução do problema inverso obtida pelo MCMC.

Para trabalhos futuros, recomenda-se aplicar outros métodos de estimação de parâmetros a fim de comparar com os resultados aqui obtidos pelo MCMC. 


\section{Agradecimentos}

Os autores agradecem à FAPERJ, Fundação Carlos Chagas Filho de Amparo à Pesquisa do Estado do Rio de Janeiro, ao CNPq, Conselho Nacional de Desenvolvimento Científico e Tecnológico, e à CAPES, Coordenação de Aperfeiçoamento de Pessoal de Nível Superior.

\section{Referências}

[1] J. V. Beck, B. Blackwell, C. R. St. Clair Jr., Inverse Heat Conduction : III-Posed Problems. Nova York : Jhon Wiley \& Sons, 1985.

[2] W. U. Costa. Técnicas Bayesianas para Engenharia Elétrica. UFMG, Minas Gerais, 2004.

[3] R. M. Cotta. Integral Transforms in Computational Heat and Fluid Flow. CRC Press, pp. 180-188, 1993.

[4] J. W. Elder. The dispersion of marked fluid in turbulent shear flow. Journal of Fluid Mechanics, Vol.5, pp. 544-560, 1959.

[5] H. B. Fischer, J. Imberger, E. J. List, R. C. Y. Koh, e N. H. Brooks. Mixing in inland and coastal waters. Elsevier, Academic Press, New York, 1979.

[6] J. Kaipio, E. Somersalo. Statistical and Computational Inverse Problems. Springer, New York, 2005.

[7] L. Pinto, A. B. Fortunato, Y. Zhang, A. Oliveira e F. E. P. Sancho. Development and validation of a three-dimensional morphodynamic modelling system for non-cohesive sediments. Ocean Modelling, vol.57, Elsevier, 2012.

[8] L. C. V. Rijn. Mathematical modeling of suspended sediment in nonuniform flows, Journal of Hydraulic Engineering, American Society of Civil Engineers, 1986.

[9] M. Schwaab e J. C. Pinto. Análise de Dados Experimentais. I. Fundamentos de Estatística e Estimação de Parâmetros. E-papers, Rio de Janeiro, Brasil, 2007.

[10] E. P. Sousa. Avaliação de Mecanismos dispersivos em rios através de problemas inversos, Dissertação de Mestrado, UERJ/IPRJ, 2009.

[11] A. L. R. Souza. Estudo do Movimento Incipiente de Sedimentos Não-Coesivos em Escoamentos com Superfície Livre, Dissertação de Mestrado, UFRJ/COPPE, 2010.

[12] A. L. R. Souza, D. C. Knupp, P. P. G. W. Rodrigues e A. J. Silva Neto. Estimativa de Parâmetros Hidrodinâmicos e Simulação do Transporte Bidimensional de Sedimentos Através da Técnica da Transformada Integral Generalizada e Inferência Bayesiana. XIX Encontro Nacional de Modelagem Computacional, João Pessoa, Paraíba, 2016. 